\title{
A comparison of infraclavicular and supraclavicular approaches to the brachial plexus using neurostimulation
}

\author{
Chun Woo Yang ${ }^{1}$, Hee Uk Kwon ${ }^{1}$, Choon-Kyu Cho ${ }^{1}$, Sung Mee Jung ${ }^{1}$, Po-Soon Kang ${ }^{1}$, Eun-Su Park ${ }^{1}$, \\ Youn Moo $\mathrm{Heo}^{2}$, and Helen Ki Shinn ${ }^{3}$
}

Departments of ${ }^{1}$ Anesthesiology and Pain Medicine, ${ }^{2}$ Orthopedic Medicine, College of Medicine, Konyang University, Daejeon, ${ }^{3}$ Department of Anesthesiology and Pain Medicine, College of Medicine, Inha University, Incheon, Korea

Background: A prospective, double blind study was performed to compare the clinical effect of vertical infraclavicular and supraclavicular brachial plexus block using a nerve stimulator for upper limb surgery.

Methods: One hundred patients receiving upper limb surgery under infraclavicular or supraclavicular brachial plexus block were enrolled in this study. The infraclavicular brachial plexus block was performed using the vertical technique with $30 \mathrm{ml}$ of $0.5 \%$ ropivacaine. The supraclavicular brachial plexus block was performed using the plumb bob technique with $30 \mathrm{ml}$ of $0.5 \%$ ropivacaine. The block performance-related pain was evaluated. This study observed which nerve type was stimulated, and scored the sensory and motor block. The quality of the block was assessed intra-operatively. The duration of the sensory and motor block as well as the complications were assessed. The patient's satisfaction with the anesthetic technique was assessed after surgery.

Results: There were no significant differences in the block performance-related pain, frequency of the stimulated nerve type, evolution of sensory and motor block quality, or the success of the block. There were no significant differences in the duration of the sensory and motor block. There was a significant difference in the incidence of Horner's syndrome. Two patients had a pneumothorax in the supraclavicular approach. There were no significant differences in the patient's satisfaction.

Conclusions: Both infraclavicular and supraclavicular brachial plexus block had similar effects. The infraclavicular approach may be preferred to the supraclavicular approach when considering the complications. (Korean J Anesthesiol 2010; 58: 260-266)

Key Words: Supraclavicular brachial plexus block, Upper limb surgery, Vertical infraclavicular brachial plexus block.

Received: December 1, 2009. Revised: December 21, 2009. Accepted: January 14, 2010.

Corresponding author: Hee Uk Kwon, M.D., Department of Anesthesiology and Pain Medicine, College of Medicine, Konyang University, 685, Gasuwon-dong, Seo-gu, Daejeon 302-718, Korea. Tel: 82-42-600-9317, Fax: 82-42-545-2132, E-mail: gangsi@kyuh.co.kr

(C) This is an open-access article distributed under the terms of the Creative Commons Attribution Non-Commercial License (http:// creativecommons.org/licenses/by-nc/3.0/), which permits unrestricted non-commercial use, distribution, and reproduction in any medium, provided the original work is properly cited. 


\section{Introduction}

There are essentially four approaches to a brachial plexus block: interscalene, supraclavicular, infraclavicular and axillary. Compared to the axillary approach, a brachial plexus block at the level of the clavicle can anesthetize all four distal upper extremity nerve territories without a requirement for a separate block of the musculocutaneous nerve.

The supraclavicular approach has an additional anatomical advantage of a blockade at a level where the brachial plexus elements are tightly grouped, which facilitates a single point injection and is believed to result in very rapid onset [1].

Anatomically, the infraclavicular approach should be feasible in almost all patients. It also has the theoretical advantages of both the supraclavicular and axillary approaches: a compact anatomical distribution of plexus structures allowing single injection of local anesthetics and a reduced risk of pneumothorax.

Both supraclavicular and infraclavicular approaches have similar distributions of anesthesia [2]. In general, proximal blocks (interscalene and supraclavicular) are believed to have faster onset than distal blocks (infraclavicular and axillary), but there is little data and consensus. To the best of our knowledge, there have been no studies comparing both approaches to the brachial plexus using nerve stimulation.

This study compared the supraclavicular and infraclavicular approaches using neurostimulation in a prospective randomized manner for a brachial plexus block in patients undergoing upper limb surgery.

\section{Materials and Methods}

After gaining approval of the Medical Ethics Committee and written informed consent from the subjects, 100 consecutive patients who were American Society of Anesthesiologists

Table 1. Demographic and Surgical Characteristics

\begin{tabular}{lcc}
\hline & Group S (n=50) & Group I (n=50) \\
\hline Age (years) & $49 \pm 18$ & $46 \pm 18$ \\
Male/Female & $24 / 26$ & $29 / 21$ \\
Height (cm) & $162 \pm 9$ & $165 \pm 8$ \\
Weight (kg) & $62 \pm 10$ & $62 \pm 12$ \\
ASA physical status (I/II/III) & $28 / 20 / 2$ & $30 / 20 / 0$ \\
Type of surgery (n) & 16 & \\
Hand & 3 & 4 \\
Wrist & 23 & 17 \\
Forearm & 8 & 9 \\
$\quad$ Elbow & $56 \pm 25$ & $61 \pm 35$ \\
Tourniquet times (min) & $65 \pm 39$ & $73 \pm 38$ \\
Duration of surgery (min) & & \\
\hline
\end{tabular}

The values are the mean \pm SD. Group S: supraclavicular brachial plexus block, Group I: infraclavicular brachial plexus block. physical status 1 to 3 , 18 years of age or older, and scheduled to undergo surgery of the elbow, forearm, or hand under brachial plexus anesthesia were prospectively included. The exclusion criteria included the following: coexisting lung, heart, liver, or kidney disease; pregnancy; inability to understand the information provided; allergy to local anesthetics; chest deformities; previous clavicle fractures; and neurological disorders. The patients were randomized to receive either a vertical infraclavicular plexus block (group I, $\mathrm{n}=50$ ) or supraclavicular plexus block (group $\mathrm{S}, \mathrm{n}=50$ ). There was no statistically significant difference in the demographical data, type of surgery, surgery time, or tourniquet time between the two groups (Table 1).

All blocks were performed by the same anesthesiologist. Standard monitoring (non-invasive blood pressure, pulse oximetry and ECG) was commenced upon arrival to the preoperative holding area. A 22-gauge 50-mm insulated stimulation short bevel needle (Stimuplex ${ }^{\circledR}$ A, B/Braun Medical, Germany) connected to a nerve stimulator (Stimuplex ${ }^{\circledR}$-DIG, B/Braun, Germany) was used for all blocks. The initial nerve stimulator settings were $1.5 \mathrm{~mA}$ with an impulse duration of $0.1 \mathrm{~ms}$. The needle position was considered to be adequate when the motor response in the hand or wrist was obtained and remained visible with a maximum current of $0.5 \mathrm{~mA}$. The local anesthetic, $30 \mathrm{ml}$ 0.5\% ropivacaine (Naropin ${ }^{\circledR}$; AstraZeneca, Sweden) was injected slowly (60 s) with intermittent aspiration.

The vertical infraclavicular approach was performed on the supine position with the upper arm along the side, but with the elbow flexed and the hand resting on the lower chest or abdomen. After identifying the landmarks, the puncture site was marked half way between the jugular notch and the most ventral part of the acromion. The needle was introduced absolutely vertical to the horizontal plane.

The supraclavicular brachial plexus block was performed according to the original procedure reported by Brown et al. [3]. The patient was placed in the supine position with their head turned toward the opposite side. The point at which the lateral border of the sternocleidomastoid muscle joins the superior aspect of the clavicle was marked, and a needle was inserted at this point in a direction that is directly posterior (perpendicular to the table). The needle was advanced until a motor response was elicited. If a motor response in the hand or wrist was not

Table 2. Incidence of Stimulated Nerve Type

\begin{tabular}{lcc}
\hline & Group S (n=50) & Group I (n=50) \\
\hline Radial nerve & $6(12)$ & $12(24)$ \\
Median nerve & $25(50)$ & $27(54)$ \\
Ulnar nerve & $19(38)$ & $11(22)$ \\
\hline
\end{tabular}

The values are the number of patients (\%). Group S: supraclavicular brachial plexus block, Group I: infraclavicular brachial plexus block. 
obtained during the initial insertion, or if the first rib was not contacted, the needle was redirected cephalad in small steps until a motor response in the hand or wrist was obtained or until it was angled approximately $30^{\circ}$. If contact with the brachial plexus was still not made, the needle was redirected caudad in small steps until a motor response was obtained or until an angle of $30^{\circ}$ caudad was reached.

Block performance-related pain was evaluated immediately after removing the needle by asking the patient to verbally quantify the level of pain using a score between 0 and 10; 0 meaning no pain and 10 meaning excruciating pain. An assessor blinded to the block technique evaluated the presence of motor and sensory blockade in each nerve territory. The sensory and motor function was assessed during the innervation of each nerve. A simultaneous comparison of the sensory and motor function in the contralateral limb was used as a point of reference. A block assessment was performed at 10 min intervals until $50 \mathrm{~min}$ after the injection.

The sensory block for each nerve (radial, median, ulnar, musculocutanoeus, and media cutaneous of forearm) of interest was performed using alcohol-soaked gauze and graded as follows: $0=$ no difference from an unblocked extremity; 1 = less cold than unblocked extremity; and 2 = no sensation of cold.

The motor block was evaluated using the forearm flexion, thumb abduction, thumb and second digit pinch and finger abduction (for the musculocuatneous, radial, median, and ulnar nerves, respectively) and scored as follows: $0=$ no loss of force; 1 = reduced force compared with the contralateral arm; and 2 = incapacity to overcome gravity.

The quality of the block was evaluated in the intraoperative time: (a) satisfactory block- surgery without patient discomfort or the need for supplementation; (b) unsatisfactory block a sensory region involved in the surgery was not completely anesthetized and the block was supplemented by the continuous infusion of propofol at $50 \mu \mathrm{g} / \mathrm{kg} / \mathrm{min}$ and sufentanil $0.1-0.3 \mu \mathrm{g} / \mathrm{kg} \mathrm{IV}$; and (c) complete failure - if the patient still experienced pain despite supplementation, general anesthesia was induced by the attending anesthesiologist using his/her preferred technique.

The duration of the sensory and motor block was assessed. The duration of the sensory block was defined as the time between the end of the local anesthetic injection and the total recovery of sensation. The duration of the motor block was defined as the time between the end of the local anesthetic injection and the total recovery of motor functions.

The side effects and complications, such as blood vessel puncture, intravascular injection, overdose, dyspnea, Horner's syndrome, and pneumothorax, were noted. The patient's satisfaction with the anesthetic technique was assessed after
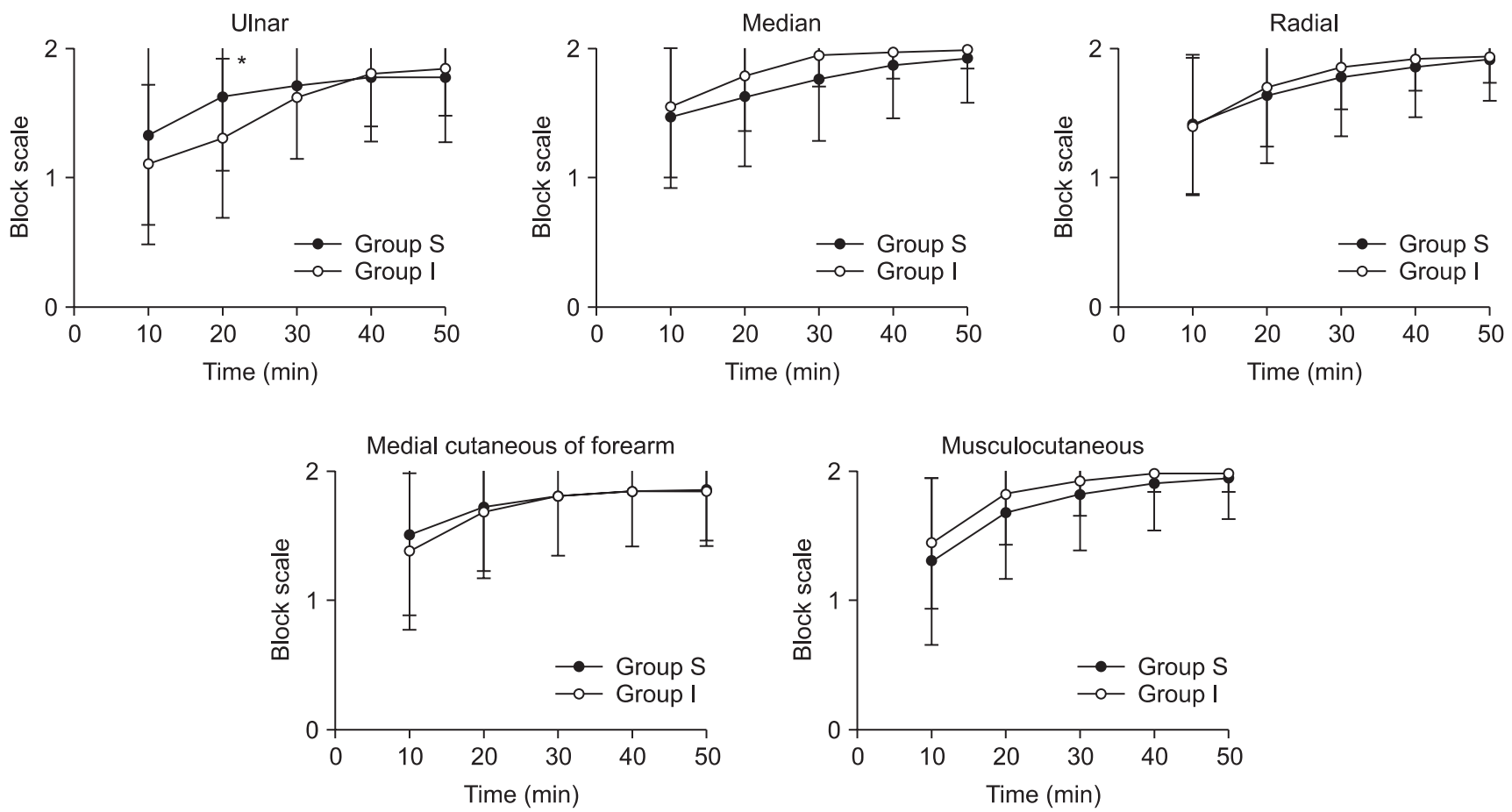

Fig. 1. Evolution of the sensory block quality in the territory of the musculocutaneous, median, radial, ulnar and medial cutaneous of the forearm nerves over the 50 minute evaluation period. Group S: supraclavicular brachial plexus block, Group I: infraclavicular brachial plexus block. ${ }^{*} \mathrm{P}<0.05$ between the groups. 
arrival in the post-anesthesia care unit using a 2-point scale $(0=$ unsatisfied; 1 = satisfied).

The values are expressed as the mean \pm SD. Group sizes (50 patients per group) were determined using the proportion sample size estimates (type 2 error $=80 \%$, type 1 error $=0.05$ ) to detect a $20 \%$ difference in the rates of complete sensory block at $50 \mathrm{~min}$. Statistical analysis was performed using a MannWhitney rank sum test, Student's t-test, Fisher's Exact test, and $\chi^{2}$ where appropriate. A P value $<0.05$ was considered significant.

\section{Results}

All 100 patients completed the study. Table 2 lists the motor responses elicited. There was no significant difference in the block related pain between group S and I, with pain scores of 3.6 \pm 1.7 and $3.2 \pm 1.5$, respectively.
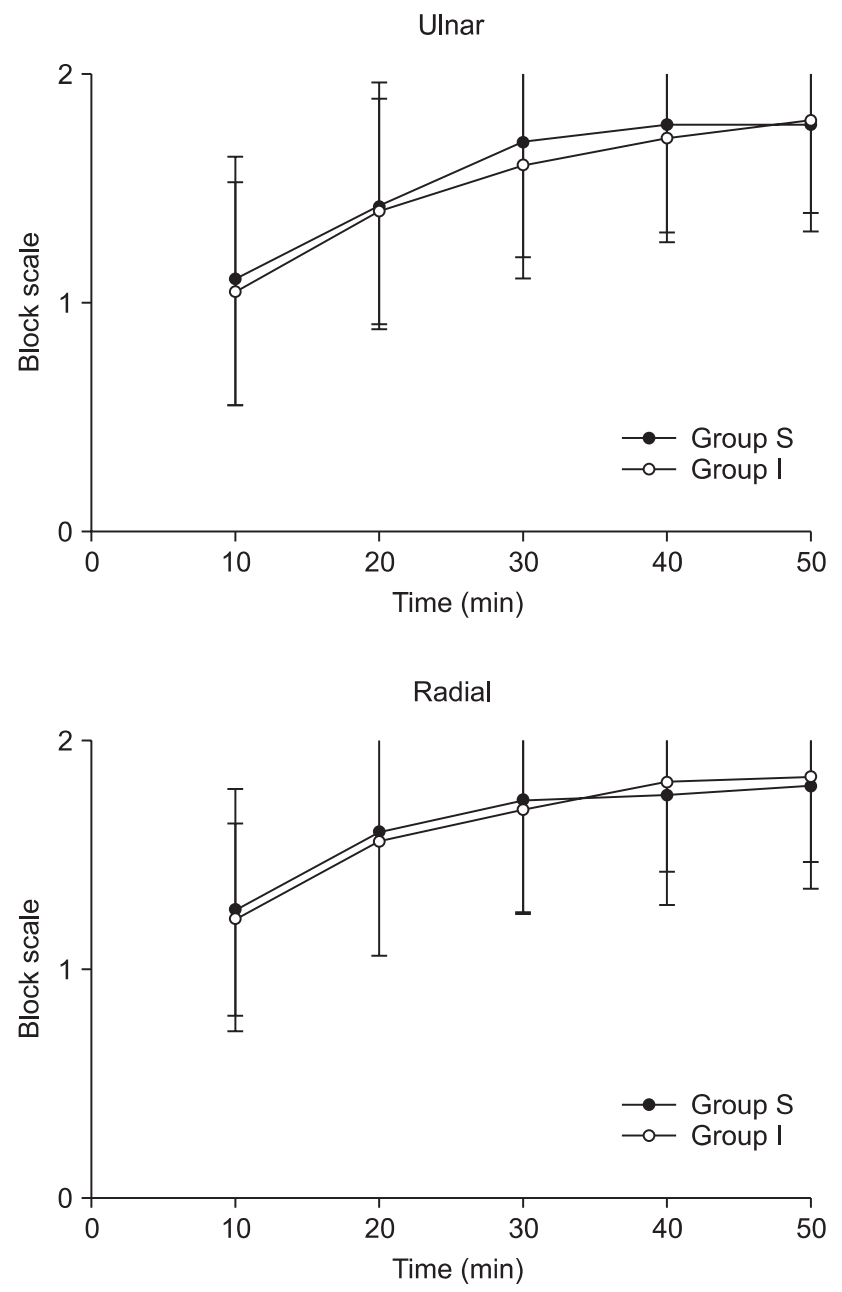

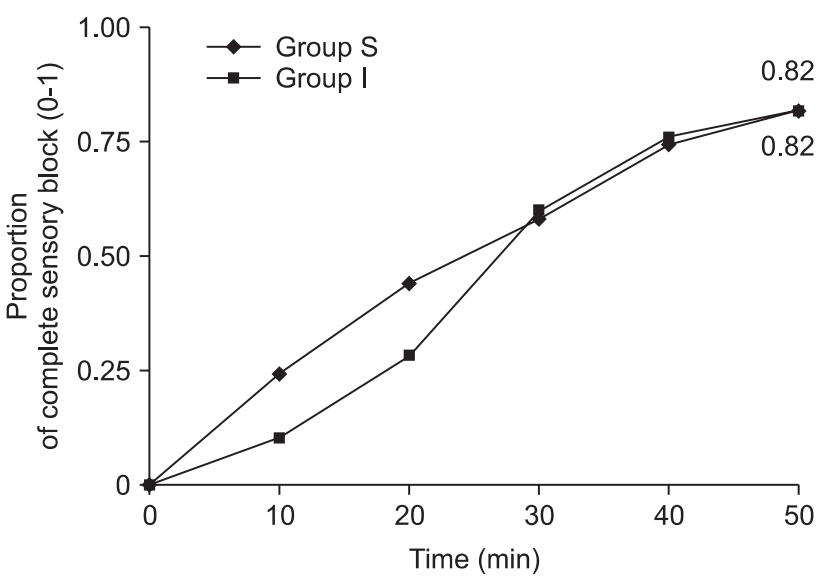

Fig. 2. Proportion of patients in each group with a complete sensory block in the musculocutanous, median, radial, ulnar and medial cutaneous of forearm territories over time. Group S: supraclavicular brachial plexus block, Group I: infraclavicular brachial plexus block.
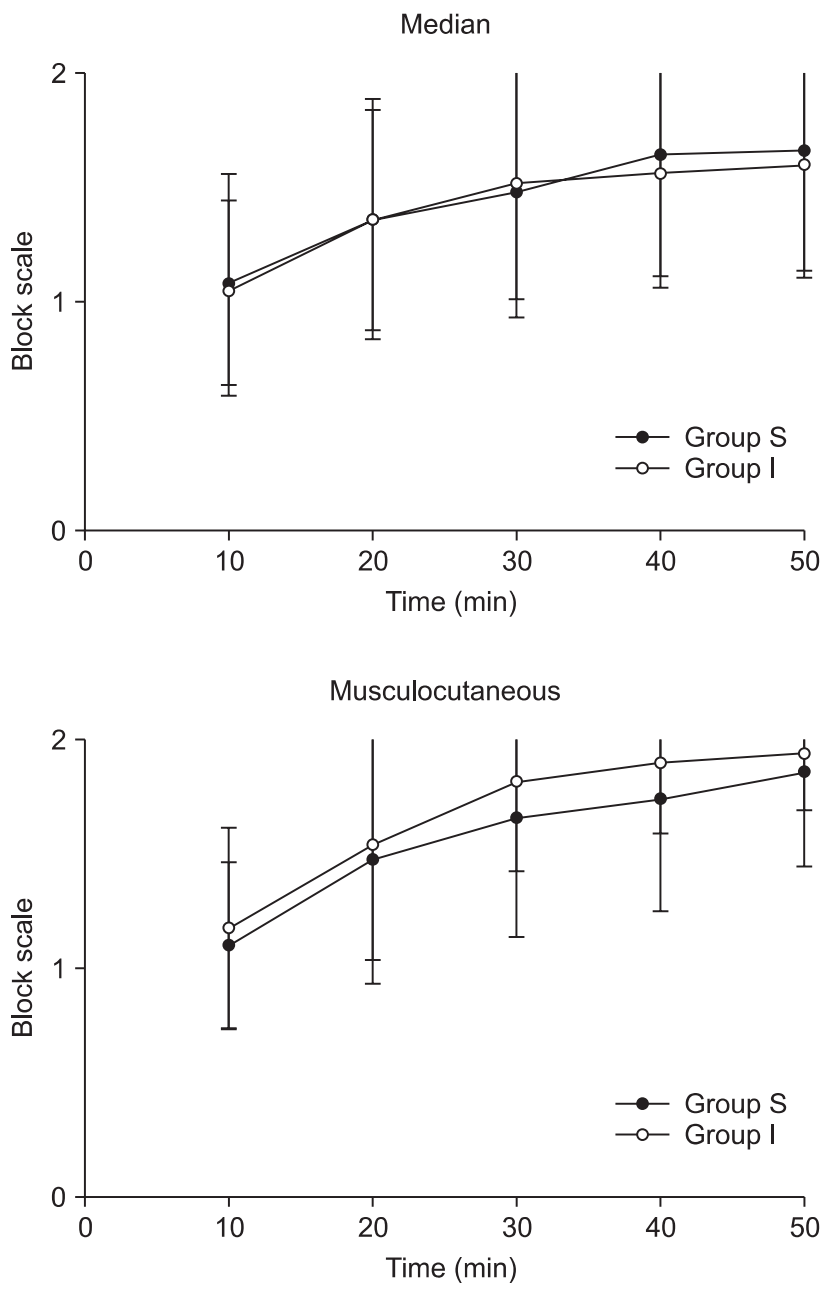

Fig. 3. Evolution of the motor block quality in the territories of the musculocutaneous, median, radial, and ulnar nerves over the 50 minute evaluation period. Group S: supraclavicualr brachial plexus block, Group I: infraclavicular brachial plexus block. 
Fig. 1 shows the progression of the sensory block according to the territory over $50 \mathrm{~min}$. There were no significant differences in the evolution of the sensory block but the sensory block was significantly better in group $\mathrm{S}$ at $20 \mathrm{~min}$ in the ulnar nerve territory $(\mathrm{P}<0.05)$. Fig. 2 shows the proportion of patients for whom complete analgesia in all territories had been achieved. There was no significant difference in the proportion of the complete sensory block over time.

The progression of the motor block (Fig. 3) paralleled that of the sensory block and there were no significant differences in the evolution of the motor block with time. There was no significant difference in the proportion of the complete motor block over time (Fig. 4).

Table 3 shows the quality of the block. A satisfactory block was achieved in 43 and 44 patients in group S and I, respectively. An unsatisfactory block was reported 6 patients in both groups. One patient in group $\mathrm{S}$ had complete failure and received a vertical infraclavicular brachial plexus block.

Table 4 lists duration of the sensory and motor block. There were no significant differences between the groups.

No systemic reactions to the local anesthetic were reported. Table 5 presents the side effects and complications. Horner's syndrome was observed in 27 and 4 patients in group S (54\%) and I (8\%), respectively. Vascular puncture while performing

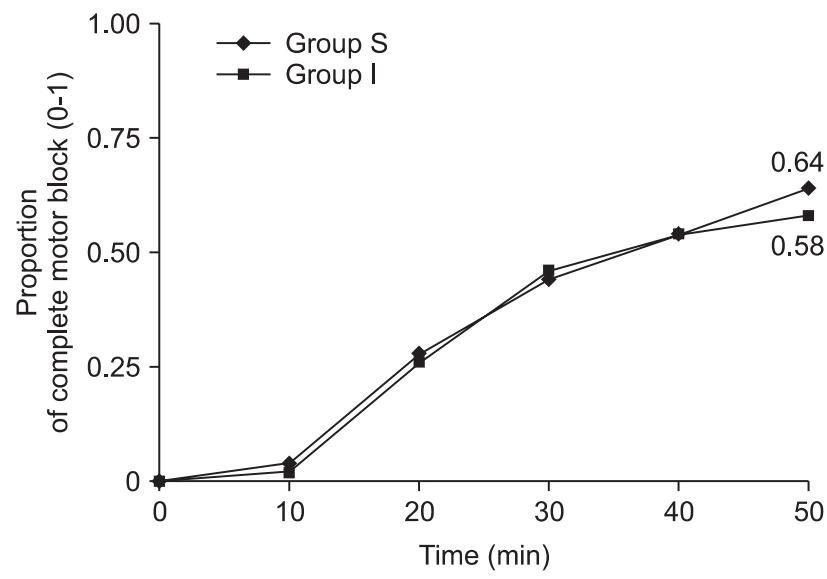

Fig. 4. Proportion of patients in each group with a complete motor block in the musculocutanous, median, radial, and ulnar territories over time. Group S: supraclavicular brachial plexus block, Group I: infraclavicular brachial plexus block.

Table 3. Quality of the Brachial Plexus Block

\begin{tabular}{lcc}
\hline & Group S (n=50) & Group I (n=50) \\
\hline Complete failure & $1(2)$ & $0(0)$ \\
Unsatisfactory block & $6(12)$ & $6(12)$ \\
Satisfactory block & $43(86)$ & $44(88)$ \\
\hline
\end{tabular}

The values are the number of patients (\%). Group S: supraclavicular brachial plexus block, Group I: infraclavicular brachial plexus block. the blocks occurred in both groups, $16 \%(\mathrm{n}=8)$ in group $\mathrm{S}$ and $14 \%(n=7)$ in group I. Three patients from group $S$ experienced mild dyspnea that was resolved after applying $6 \mathrm{~L}$ of oxygen by a mask. A pneumothorax was observed in 2 patients in group $\mathrm{S}$ (4\%), but none in group I. A thoracostomy tube was not placed.

Table 6 shows the patient's satisfaction. There were no significant differences in the level of patient's satisfaction between the groups. Two of the 97 patients were unsatisfied: one in group S and one group I. One patient in group S had a pneumothorax after the block, and one patient in the group I was unhappy with the prolonged sensory and motor block with ropivacaine.

\section{Discussion}

In this study, the supraclavicular and infraclavicular approach to the brachial plexus using neurostimulation showed no important clinical differences, except for the high incidence of Horner's syndrome and the pneumothorax in 2 patients with the supraclavicular approach.

A brachial plexus block can be performed using several approaches. Selection of the preferred approach is determined by the innervations of the surgical site, risk of regional anesthesia-related complications, as well as the preference

Table 4. Duration of the Sensory and Motor Block

\begin{tabular}{lcc}
\hline & Group S (n=47) & Group I (n=49) \\
\hline Sensory block (min) & $763 \pm 202$ & $827 \pm 175$ \\
Motor block (min) & $774 \pm 231$ & $828 \pm 210$ \\
\hline
\end{tabular}

The values are the mean \pm SD. Group S: supraclavicular brachial plexus block, Group I: infraclavicular brachial plexus block.

Table 5. Side Effects and Complications

\begin{tabular}{lcc}
\hline & Group S (n=50) & Group I (n=50) \\
\hline Horner syndrome* & $27(54)$ & $4(8)$ \\
Dyspnea & $3(6)$ & $0(0)$ \\
Pneumothorax & $2(4)$ & $0(0)$ \\
Vascular puncture & $8(16)$ & $7(14)$ \\
\hline
\end{tabular}

The values are the number of patients (\%). Group S: supraclavicular brachial plexus block, Group I: infraclavicular brachial plexus block. $* \mathrm{P}<0.05$ between the groups.

Table 6. Patient's Satisfaction

\begin{tabular}{lcc}
\hline & Group S (n=48) & Group I ( $\mathrm{n}=49)$ \\
\hline Unsatisfied & $1(2)$ & $1(2)$ \\
Satisfied & $47(98)$ & $48(98)$ \\
\hline
\end{tabular}

The values are the number of patients (\%). Group S: supraclavicular brachial plexus block, Group I: infraclavicular brachial plexus block. 
and experience of the anesthesiologist. Other factors may be considered, such as the reliability, ease and rapidity, and patient comfort during block performance.

Historically, the supraclavicular approach to the brachial plexus can provide excellent anesthesia for upper-extremity surgery. Compared with the axillary block, the supraclavicular approach to the brachial plexus offers a distinct advantage, particularly a faster onset of a dense block with a single injection using less local anesthesia [4]. However, many anesthetists prefer not to perform this technique for fear of causing a pneumothorax. The supraclavicular approach using the plumbbob technique was also selected to avoid pneumothorax.

In this study, both approaches showed similar results in terms of complete sensory block rates and quality of block. At $50 \mathrm{~min}$, complete analgesia of all territories was achieved in $82 \%$ ( $\mathrm{n}=$ $41)$ and in $82 \%(n=41)$ of patients in group I and S, respectively. Sufficient surgical analgesia in the vertical infraclavicular approach was reported by Kilka et al. [5] in 95\% of patients at $30 \mathrm{~min}$ using $40 \mathrm{ml}$ of prilocaine $1.5 \%$ and $10 \mathrm{ml}$ of bupivacaine $0.5 \%$. Neuburger et al. [6], without specifying the time of assessments, reported sufficient surgical anesthesia in $87 \%$ and $88 \%$ of patients. In the supraclavicular block, Franco et al. [1] reported a $97.2 \%$ success rate using the subclavian perivascular technique in 1,001 patients. Possible reasons for the lower success rate observed in both groups include the lower volume of local anesthesia used, operator's inexperience, different local anesthetics used or the definition of success.

In this study, the ulnar $(n=9)$ and medial cutaneous of the forearm nerve $(n=6)$ were frequently incompletely blocked in group S. The intimate relationship of the lower trunk to the subclavian artery might be one reason why better results are obtained when a trunk other than the superior trunk is made the epicenter of the injection [7]. It is possible that depositing the local anesthetic close to the lower trunk will increase the likelihood of blocking it directly, thereby overcoming the obstacle to diffusion that the closely located pulsatile artery might exert on this trunk. However, this remains speculative and will require more study. Interestingly, the ulnar $(n=8)$ and medial cutaneous of the forearm nerve $(n=7)$ were also incompletely blocked in group I. This might be explained by the anatomical barrier [8].

There are no reports comparing the supraclavicular with infraclavicular approach using neurostimulation. Several studies compared the supraclavicular approach with the infraclavicular approach with ultrasound. Arcand et al. [9] compared ultrasound-guided supraclavicular with infraclavicular blocks and reported no significant difference in either the block performance or onset times or block efficacy. In contrast, Koscielniak et al. [10] reported that an ultrasoundguided infraclavicular block had a faster onset, better surgical efficacy and fewer adverse events than a supraclavicular block. Recently, Fredrickson et al. [11] compared an ultrasoundguided supraclavicular block using multiple injection with ultrasound-guided triple injection infraclavicular block. They reported that the corner pocket supraclavicular and infraclavicular brachial plexus block were associated with similar onset times and sensory blockade at $30 \mathrm{~min}$. In addition, surgical anesthesia success was higher in the patients receiving an infraclavicular block as a result of the more complete blockade in the distribution of the ulnar nerve.

The incidence of vessel puncture was similar in both groups. None of them resulted in serious complications, such as seizures or hematoma. This might be due to the slow injection technique with repeated aspiration and the use of atraumatic needles.

The diaphragmatic function was examined in this study. The incidence of phrenic nerve block appears to be similar to the incidence of Horner's syndrome because the phrenic nerve is situated on the belly of the anterior scalene muscle and the cervical sympathetic chain medial. According to Rettig et al. [12], Horner's syndrome is a clinically significant sign (100\%) that predicts changes in hemidiaphragmatic movement. However, in their patients, changes in hemidiaphragmatic movement were also observed without Horner's syndrome. In this study, Horner's syndrome was encountered in 27 and 4 patients in group S (54\%) and group I (8\%), respectively. When the complication rates between the supraclavicular and infraclavicular approaches are compared, an impairment in diaphragmatic movements can be rated as $100 \%$ for interscalene [13], $50 \%$ to $77 \%$ for supraclavicular [14,15], $24 \%$ to $26 \%$ for proximal infraclavicular [12], and $0 \%$ for more distal infraclavicular blocks $[16,17]$.

In this study, pneumothorax occurred in two patients with supraclavicular approach but in none using infraclavicular approach. A pneumothorax is a serious complication associated with the supraclavicular approach. This has also been reported after interscalene [18], coracoid and vertical infraclavicular blocks $[19,20]$. The reported incidence of pneumothorax after a supraclavicular block is $0.5 \%$ to $6.1 \%$. The plumb-bob and subclavian perivascular approaches were designed in part to reduce the risk of pneumothorax. The risk of pneumothorax in tall, thin patients might be reduced further by initially directing the needle $45^{\circ}$ cephalad during the supine plumb-bob technique, rather than directly toward the floor. This magnetic resonance imaging finding has not been confirmed clinically. The incidence of pneumothorax is likely to be reduced by the operator's experience, using shorter needles, and taking extra care with tall, thin patients who are more likely to have high apical pleural reflections or in patients with emphysema. An ultrasound-guided supraclavicular block might reduce the risk 
of pneumothorax because the pleura and first rib are often easy to visualize. Perlas et al. [21] reported that an ultrasound-guided supraclavicular block is associated with a high success rate and low complication rate with no pneumothorax in a series of 510 consecutive patients.

This study had some limitations. First, a single anesthesiologist performed all the blocks. Although this eliminates the interoperator variability, it might limit generalizing the results. There was more experience with the infraclavicular approach than with supraclavicular approach at that time, which might produce more complications in the supraclavicular approach. Second, the block performance time, onset time and time of readiness for surgery were not assessed. These are important factors when two different approaches to the brachial plexus are compared. Third, two out of seven terminal nerves (axillary, medial brachial cutaneous) were not used. Many anesthesiologists consider anesthesia of these two nerves to be necessary for surgery of the elbow and for the patients' tolerance of the pneumatic arm tourniquet. Fourth, in the supraclavicular approach, the type of response (identification of a specific nerve) is an important factor that influences the overall success. Stimulation of the middle trunk (hand contraction or paresthesia) is associated with higher success rates [7]. In this study, most of the elicited motor responses in supraclavicular approach were the median or ulnar nerve types.

In conclusion, these results suggest that both the supraclavicular and infraclavicular approach to the brachia plexus had similar clinical efficacy but the supraclavicular block caused a pneumothorax. These results suggest that the infraclavicular approach might be preferable for hand, forearm, and/or elbow surgery. However, more large scale studies will be needed to compare the supraclavicular block with the infraclavicular block using neurostimulation.

\section{References}

1. Franco CD, Vieira ZE. 1,001 subclavian perivascular brachial plexus blocks: success with a nerve stimulator. Reg Anesth Pain Med 2000; 25: 41-6.

2. Neal JM, Gerancher JC, Hebl JR, Ilfeld BM, McCartney CJ, Franco $\mathrm{CD}$, et al. Upper extremity regional anesthesia: essentials of our current understanding, 2008. Reg Anesth Pain Med 2009; 34: 13470.

3. Brown DL, Cahill DR, Bridenbaugh LD. Supraclavicular nerve block: anatomic analysis of a method to prevent pneumothorax. Anesth Analg 1993; 76: 530-4.

4. Brown AR. Anaesthesia for procedures of the hand and elbow. Best Pract Res Clin Anaesthesiol 2002; 16: 227-46.

5. Kilka HG, Geiger P, Mehrkens HH. Infraclavicular vertical brachial plexus blockade. A new method for anesthesia of the upper extremity. An anatomical and clinical study. Anaesthesist 1995; 44:
339-44.

6. Neuburger M, Kaiser H, Rembold-Schuster I, Landes H. Vertical infraclavicular brachial-plexus blockade. A clinical study of reliability of a new method for plexus anesthesia of the upper extremity. Anaesthesist 1998; 47: 595-9.

7. Hickey R, Garland TA, Ramamurthy S. Subclavian perivascular block: influence of location of paresthesia. Anesth Analg 1989; 68: 767-71.

8. Dolan J. Fascial planes inhibiting the spread of local anesthetic during ultrasound-guided infraclavicular brachial plexus block are not limited to the posterior aspect of the axillary artery. Reg Anesth Pain Med 2009; 34: 612-3.

9. Arcand G, Williams SR, Chouinard P, Boudreault D, Harris P, Ruel $\mathrm{M}$, et al. Ultrasound-guided infraclavicular versus supraclavicular block. Anesth Analg 2005; 101: 886-90.

10. Koscielniak-Nielsen ZJ, Frederiksen BS, Rasmussen H, Hesselbjerg L. A comparison of ultrasound-guided supraclavicular and infraclavicular blocks for upper extremity surgery. Acta Anaesthesiol Scand 2009; 53: 620-6.

11. Fredrickson MJ, Patel A, Young S, Chinchanwala S. Speed of onset of 'corner pocket supraclavicular' and infraclavicular ultrasound guided brachial plexus block: a randomised observer-blinded comparison. Anaesthesia 2009; 64: 738-44.

12. Rettig HC, Gielen MJ, Boersma E, Klein J, Groen GJ. Vertical infraclavicular block of the brachial plexus: effects on hemidiaphragmatic movement and ventilatory function. Reg Anesth Pain Med 2005; 30: 529-35.

13. Urmey WF, Talts KH, Sharrock NE. One hundred percent incidence of hemidiaphragmatic paresis associated with interscalene brachial plexus anesthesia as diagnosed by ultrasonography. Anesth Analg 1991; 72: 498-503.

14. Neal JM, Moore JM, Kopacz DJ, Liu SS, Kramer DJ, Plorde JJ. Quantitative analysis of respiratory, motor, and sensory function after supraclavicular block. Anesth Analg 1998; 86: 1239-44.

15. Mak PH, Irwin MG, Ooi CG, Chow BF. Incidence of diaphragmatic paralysis following supraclavicular brachial plexus block and its effect on pulmonary function. Anaesthesia 2001; 56: 352-6.

16. Rodriguez J, Barcena M, Rodriguez V, Aneiros F, Alvarez J. Infraclavicular brachial plexus block effects on respiratory function and extent of the block. Reg Anesth Pain Med 1998; 23: 564-8.

17. Dullenkopf A, Blumenthal S, Theodorou P, Roos J, Perschak H, Borgeat A. Diaphragmatic excursion and respiratory function after the modified Raj technique of the infraclavicular plexus block. Reg Anesth Pain Med 2004; 29: 110-4.

18. Borgeat A, Ekatodramis G, Kalberer F, Benz C. Acute and nonacute complications associated with interscalene block and shoulder surgery: a prospective study. Anesthesiology 2001; 95: 875-80.

19. Desroches J. The infraclavicular brachial plexus block by the coracoid approach is clinically effective: an observational study of 150 patients. Can J Anaesth 2003; 50: 253-7.

20. Neuburger M, Landes H, Kaiser H. Pneumothorax in vertical infraclavicular block of the brachial plexus. Review of a rare complication. Anaesthesist 2000; 49: 901-4.

21. Perlas A, Lobo G, Lo N, Brull R, Chan VW, Karkhanis R. Ultrasoundguided supraclavicular block: outcome of 510 consecutive cases. Reg Anesth Pain Med 2009; 34: 171-6. 